\title{
Antimicrobial resistance: Implications for therapy of infections with common childhood pathogens
}

\author{
DAVID P SPEERT MD FRCPC
}

$\mathrm{T}$ herapy of infectious diseases caused by bacteria resistant to multiple antimicrobial agents has emerged as one of the greatest challenges facing clinicians. Problems created by antibiotic resistance have profound effects upon the practice of pediatrics, resulting in a continuing need for revision of recommended therapies of common infectious diseases. For example, many pediatricians remember when penicillin $\mathrm{G}$ was the drug of choice for Staphylococcus aureus and ampicillin monotherapy was recommended as the initial therapy for children with bacterial meningitis. With the inappropriate use of antimicrobial agents for treatment of conditions such as the common cold, substantial changes have occurred in microbial resistance patterns. Furthermore, with widespread international travel and increased importation of food, spread of antibiotic-resistant bacteria has been promoted between developed and developing countries. Bacterial antibiotic resistance has evolved inevitably, forcing physicians to abandon 'conventional' therapeutic agents for others that often have a broader spectrum of activity, greater expense and higher toxicity. In light of the dramatic changes witnessed over the past several years, this review is written to provide clinicians with guidelines for therapy of some common childhood infections and to outline measures that may prevent spread and acquisition of antibiotic-resistant organisms.

Division of Infectious and Immunological Diseases, Department of Pediatrics, University of British Columbia and British Columbia's Children's Hospital, Vancouver, British Columbia

Correspondence: Dr David P Speert, Room 303, Research Centre, 950 West 28 th Avenue, Vancouver, British Columbia V5Z 4 H4. Telephone 604-875-2438, fax 604-875-2226, e-mail speert@cbdn.ca This statement is also being published in Paediatrics \& Child Health 1996;1(1):49-53.

\section{MECHANISMS OF ANTIMICROBIAL RESISTANCE}

Bacteria are remarkably resourceful and adaptable in their capacity to resist a wide array of antimicrobial agents. Various mechanisms of resistance may have evolved in response to pressures applied by 'natural' antibiotics produced by other microorganisms with which these bacteria coexist. Resistance has been further induced under the pressure imposed by antimicrobial therapy and by effects of antibiotics added to animal feeds. It appears that acquisition of antimicrobial resistance is not linked to enhanced bacterial virulence. Most currently recognized antimicrobial resistance mechanisms can be classified into one of the following categories (Table 1):

1. Altered target site for the antimicrobial agent;

2. Enzymatic inactivation of the antimicrobial agent;

3. Decreased permeability of the bacterial envelope.

Resistance to antimicrobial agents is encoded genetically, either stably within the bacterial chromosome or on mobile extra chromosomal units (plasmids or transposons) that have the ability to move from one type of bacterium to another. Plasmid-mediated transfer of genetic material can occur by several different processes, resulting in spread of resistance to one or more antimicrobial agents among bacteria of the same or different species and genus. For instance, resistance can be transmitted between Gram-positive and Gram-negative bacteria. The following factors may enhance the likelihood of acquiring an infection with bacteria resistant to first-line antimicrobial agents:

1. Recent or current antimicrobial therapy for the same or a different infection including recent suboptimal antimicrobial therapy (inadequate duration, dosage or combination of drugs); 
TABLE 1

Mechanisms of bacterial resistance to antimicrobial agents

\begin{tabular}{llll}
\hline \multirow{2}{*}{$\begin{array}{l}\text { General mechanism of } \\
\text { resistance }\end{array}$} & Bacterial species & Antimicrobial agent & Specific mechanism \\
\cline { 2 - 4 } & Streptococcus pneumoniae & Penicillin & Altered penicillin-binding protein \\
Altered target site & Staphylococcus aureus & Penicillin & Beta-lactamase production \\
Enzymatic inactivation & Gram-negative bacilli & Aminoglycosides & Aminoglycoside inactivating enzymes \\
& Pseudomonas aeruginosa & Gentamicin & $\begin{array}{c}\text { Decreased porin-mediated } \\
\text { penetration through outer membrane }\end{array}$ \\
\hline
\end{tabular}

TABLE 2

Alternative antimicrobial agents for selected bacterial pathogens resistant to drugs of first choice

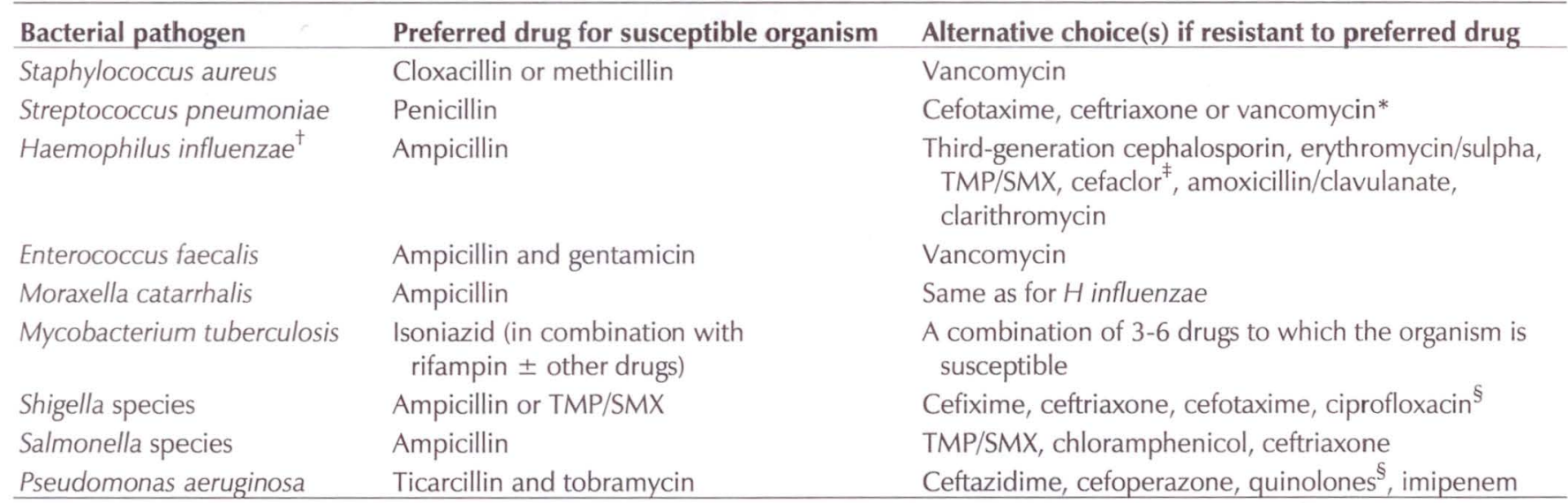

${ }^{*}$ Choice determined by site of infection and in vitro antimicrobial resistance results; ${ }^{\dagger}$ Infection amenable to oral therapy; ${ }^{\ddagger}$ Or other oral second- or third-generation cephalosporin; ${ }^{\S}$ Not approved for use in children. TMP/SMX Trimethoprim/sulfamethoxazole

2. Hospitalization, particularly in a tertiary care teaching hospital as opposed to a community hospital (nosocomial infection);

3. Attendance at a facility where crowding occurs and transmission of organisms is facilitated, such as child care centres, nursing homes and homes for the physically or mentally challenged;

4. Recent travel to a country in the Third World, where resistance rates tend to be high;

5. Shifts in social behaviour that accelerate disease transmission and increase antibiotic requirements;

6. Hospitalization outside Canada.

\section{SPECIFIC PROBLEMS OF ANTIMICROBIAL RESISTANCE IN PEDIATRICS}

Table 2 illustrates several bacterial pathogens that have developed resistance to antimicrobial agents of first choice. The list is by no means exhaustive; it is designed to provide guidance to the clinician faced with one of the more common situations where the first-line drug of choice cannot be used. Space does not permit a discussion of each of these problems; following is a brief review of several situations where the choice of antimicrobial therapy has been altered by recent changes in resistance patterns.
Invasive infection with Streptococcus pneumoniae: Penicillin has long been the therapeutic agent of choice for infections with $S$ pneumoniae. However, over the past decade, the incidence of infections with penicillin resistant strains has risen; lately this has been coupled with resistance to third-generation cephalosporins. This change in susceptibility of $S$ pneumoniae to beta-lactam antimicrobial agents has created a major challenge in the therapy of invasive infections by this common pediatric pathogen. Resistance is mediated by changes in penicillin-binding proteins of the bacterial cell wall to which the antibiotic normally binds and kills the bacterium.

Resistance of $S$ pneumoniae to penicillin is considered to be intermediate if the minimal inhibitory concentration (MIC) is 0.1 to $1.0 \mu \mathrm{g} / \mathrm{mL}$ and high level if the MIC is greater than $1.0 \mu \mathrm{g} / \mathrm{mL}$. Pneumococci are intermediately resistant to cefotaxime and ceftriaxone if the MIC is 0.5 to $1.0 \mu \mathrm{g} / \mathrm{mL}$ and highly resistant if the MIC is greater than $1.0 \mu \mathrm{g} / \mathrm{mL}$. The distinction is of practical importance because infection with intermediately resistant strains usually can be cured with beta-lactam antibiotics if the infection is at a body site where the antibiotic is able to penetrate to reach concentrations substantially in excess of the MIC. However, meningitis with $S$ pneumoniae intermediately resistant to penicillin and cephalosporins should not be treated with these agents because bactericidal concentrations of the drug in the cerebrospinal fluid (CSF) may not be attained. Fortunately, all pneumococcal 
strains that are resistant to penicillin and/or cephalosporins currently are susceptible to vancomycin.

Although antimicrobial disk susceptibility testing provides a good first estimation of resistance patterns, MIC should be determined for all $S$ pneumoniae isolates from normally sterile body sites (including CSF, blood, pleural and synovial fluid) deemed to be resistant by disk susceptibility. It is essential that isolates be tested for susceptibility to penicillin and thirdgeneration cephalosporins. If the pneumococcus is resistant to all tested beta-lactam agents, susceptibility to vancomycin, imipenem and rifampin should be assessed by MIC determination.

Therapy of antimicrobial-resistant pneumococci is determined by the site of the infection and the specific nature of the susceptibility pattern. The following principles should aid in the choice of an appropriate regimen:

1. The susceptibility of the $S$ pneumoniae strain should be determined. If the strain is resistant to penicillin, susceptibility to cefotaxime, ceftriaxone, vancomycin and rifampin should be determined.

2. If the strain is susceptible to penicillin (MIC less than $0.1 \mu \mathrm{g} / \mathrm{mL}$ ) the patient should be treated with penicillin.

3. If the infection does not involve the central nervous system (CNS) and the strain is intermediately resistant to penicillin, either penicillin or another drug to which the strain is susceptible (cefotaxime, ceftriaxone or vancomycin) may be used. Rifampin should never be used as a single agent since resistance may develop during therapy.

4. For empirical therapy of a confirmed or highly suspected pneumococcal CNS infection before notification of susceptibility test results, a combination of vancomycin and cefotaxime or ceftriaxone is recommended. Therapy should subsequently be adjusted based on the results of MIC testing.

5. If the infection involves the CNS, penicillin should be used only if the pneumococcal isolate is susceptible. If the strain is intermediately resistant, an alternative drug should be chosen based on susceptibility testing results (MIC). Cefotaxime or ceftriaxone should be used only if the strain is susceptible to it.

6. For pneumococcal infection of the CNS with a penicillinresistant strain, cefotaxime or ceftriaxone should be used if the isolate is susceptible to it. If the strain is resistant to penicillin and cephalosporins, the recommended regimen is vancomycin ( $60 \mathrm{mg} / \mathrm{kg} /$ day) with or without oral rifampin. Rifampin penetrates well into the CSF, thereby complementing vancomycin, an agent with unpredictable penetration.

Methicillin resistant $S$ aureus: $S$ aureus strains resistant to methicillin and cloxacillin pose a particularly serious problem because they can be transmitted from patient to patient in hospitals; indeed, most strains appear to be acquired noso- comially. Resistance (like that of the pneumococcus to penicillin) is mediated by a modification in the penicillin-binding protein to which methicillin normally binds. Methicillin-resistant $S$ aureus strains also are resistant to cephalosporins regardless of in vitro susceptibility testing results. Strains resistant to methicillin are also often resistant to erythromycin, clindamycin, aminoglycosides, tetracycline and chloramphenicol. Intravenous vancomycin is the drug of choice for such infections. Infections should be recognized and treated, and infection control precautions instituted as early as possible, to prevent nosocomial spread.

Group A streptococcus: Group A streptococci are uniformly susceptible to penicillin, an agent that continues to be the drug of choice. However, in patients unable to receive penicillin because of allergy, alternative agents are required. Erythromycin has been the drug of choice for therapy of streptococcal infections of the throat and skin in patients allergic to penicillin, but resistance occurs. Vancomycin or clindamycin is the recommended agent for erythromycin-resistant invasive group A streptococcal infections in patients allergic to penicillin.

Haemophilus influenzae: H influenzae type b was the leading cause of meningitis in children before the recent introduction of universal haemophilus immunization in infancy. Nonetheless, infections with type b strains continue to occur sporadically, and nontypeable $H$ influenzae is a major cause of bacterial otitis media. In most cases, $H$ influenzae resistance to ampicillin is mediated by beta-lactamase production, but a small percentage of strains owe their resistance to an altered penicillin-binding protein.

For invasive ampicillin-resistant $H$ influenzae infections, a third-generation cephalosporin, such as cefotaxime or ceftriaxone, is the drug of choice. For noninvasive infections, such as otitis media or sinusitis, a range of antimicrobial options is available (Table 2). On the basis of efficacy, there is little evidence available that any of these alternatives is superior to the others. The choice among beta-lactamase-resistant oral antibiotics should therefore be made on the basis of patient acceptance (taste, dosing interval and price) and the rate of adverse effects.

Moraxella catarrhalis: $M$ catarrhalis is an important cause of respiratory tract infection, and accounts for approximately $10 \%$ of cases of otitis media. Whereas ampicillin is the drug of choice for otitis media, over $75 \%$ of $M$ catarrhalis produces beta-lactamase rendering it resistant to ampicillin. Although the $M$ catarrhalis beta-lactamase is distinct from that produced by $H$ influenzae, the antimicrobial agents effective against both organisms are the same. The choice of one agent over the others should be guided as described above for H influenzae. Escherichia coli: $E$ coli is an important cause of infection in childhood (eg, urinary tract infection and neonatal sepsis/meningitis). Strains of $E$ coli may be broadly susceptible or resistant to a wide range of antimicrobial agents. Therapy should be directed by in vitro antimicrobial susceptibility results.

Shigella species: Antimicrobial therapy for shigellosis is recommended to shorten the duration of disease and eliminate bacterial fecal shedding in acute infection. Trimethoprim/sulfamethoxazole have been the agents of choice for shigella 
gastroenteritis, but resistance has emerged in a large percentage of isolates from both the Third World and Canada. Shigellosis should be treated with ampicillin or trimethoprim/ sulfamethoxazole if the infecting organism is susceptible; in areas where resistance to both antibiotics is common, initial therapy with another agent (such as cefixime) should be considered. Therapy of persons infected with resistant strains should be guided by the in vitro antimicrobial susceptibility pattern. Candidate antimicrobial agents include cefixime, ceftriaxone, cefotaxime, ciprofloxacin or ofloxacin; the latter two are not approved for use in children and should therefore only be used with caution.

\section{MEASURES TO LIMIT THE SPREAD OF ANTIMICROBIAL RESISTANCE}

Antimicrobial resistance to a wide array of drugs has developed at an alarming rate over the past decade. Acquisition of resistance has resulted from promiscuous antimicrobial prescribing practices, nosocomial transmission of resistant organisms and changes in societal conditions (eg, increased use of child care centres and urban crowding). In most cases, drugs are available to treat infections with antibiotic-resistant strains of bacteria. However, examples of impossible therapeutic challenges have already appeared (eg, multiply resistant Mycobacterium tuberculosis, vancomycin-resistant enterococci) or are imminent (eg, vancomycin-resistant $S$ aureus).

To limit the spread of antimicrobial resistance among pathogens that infect children, a number of strategies should be adopted or more strongly encouraged, as follows:

1. Antimicrobial therapy should be limited to those situations where there is a clear indication and should be administered for the shortest effective duration. The prescription of oral antibiotics for viral respiratory tract infections should be discouraged.

2. Documented infections should be treated with the appropriate dose, duration and narrowest spectrum antimicrobial agent recommended.

3. Prophylactic antibiotic therapy should be given only when indicated and for no longer than the recommended duration.

\section{ANNOTATED RECOMMENDED READING}

1. Dever LA, Dermody TS. Mechanisms of bacterial resistance to antibiotics. Arch Intern Med 1991;151:886-95.

This paper describes the molecular mechanisms for the three major classes of antimicrobial resistance.

2. Friedland IR, McCracken GH. Management of infections caused by antibiotic-resistant Streptococcus pneumoniae. N Engl J Med 1994;331:377-82.

This paper provides an excellent overview of the problem of penicillin-resistant pneumococci, with recommendations for therapy of meningitis, sepsis, pneumonia and otitis media.

3. Iseman MD. Treatment of multidrug-resistant tuberculosis. N Engl J Med 1993;329:784-91.

This paper provides an overview of the problem of multidrugresistant Mycobacterium tuberculosis and recommendations for therapy.

4. Kunin CM. Resistance to antimicrobial drugs - a worldwide calamity. Ann Intern Med 1993;118:557-61.

This paper provides a historical overview of antimicrobial
4. Empirical antibiotic therapy should be limited to situations where there are clear indications and for the shortest appropriate duration.

5. Universal immunization of infants and children should be vigorously promoted to prevent infection with bacteria that have a great potential for antimicrobial resistance (eg, H influenzae type b).

6. The use of antimicrobial agents that provide the only option for cure of resistant bacterial infections should be limited. For instance, vancomycin use in tertiary care hospitals should be reserved for situations where no alternatives are available.

7. Measures should be taken to maintain sanitation in environments where bacteria are likely to be spread (such as child care centres).

8. In hospitals, strict barrier precautions and other infection control measures should be enforced to limit the spread of antimicrobial resistant pathogens, such as methicillinresistant $S$ aureus.

9. The marketing of over-the-counter antimicrobial agents for both human and animal use should be discouraged.

\section{ANTIMICROBIAL THERAPY DURING UNCERTAIN TIMES}

In the present environment of rapidly changing microbial ecology, no assumptions about antimicrobial susceptibility patterns of pediatric pathogens are secure. Even bacterial species that have been susceptible to penicillin (such as streptococci and neisseria) can change, rendering the first-line therapy imperfect. Clinicians should keep the spectre of antimicrobial resistance in mind when dealing with both common and unusual pathogens and should be quick to respond when conventional therapy fails to provide a cure. In this era of evolving antimicrobial resistance, the clinician must document significant infections with culture and respond to the susceptibility profile provided by the clinical microbiology laboratory. With close cooperation among clinicians, microbiologists and infection control officers, the perils of antimicrobial resistance can be minimized.

resistance, a global perspective on the problem and strategies for curbing its spread.

5. Leggiadro RJ. Penicillin- and cephalosporin-resistant Streptococcus pneumoniae: an emerging microbial threat. Pediatr Infect Dis J 1994;93:500-3.

This paper provides laboratory standards for determining penicillin resistance in pneumococci and recommendations for therapy of presumed or proven pneumococcal infections in children.

6. Murray BE. New aspects of antimicrobial resistance and the resulting therapeutic dilemmas. J Infect Dis 1991;163:1185-94. This paper provides an overview of the mechanisms of antibiotic resistance and recommended approaches to therapy of resistant staphylococci, streptococci, neisseria and enterococci.

7. Smith AL. Antibiotic resistance in pediatric pathogens. Infect Dis Clin North Am 1992;6:177-95.

This paper provides a comprehensive review of antimicrobial resistance of pathogens with which children are infected. It describes the genetic basis for antimicrobial resistance and describes each of the problematic pediatric pathogens - group A 
streptococcus, $S$ pneumoniae, group B streptococcus, $S$ aureus, Staphylococcus epidermidis, Neisseria meningitidis,

$M$ catarrhalis and $H$ influenzae.

8. Swartz MN. Hospital-acquired infections: diseases with increasingly limited therapies. Proc Natl Acad Sci USA 1994;91:2420-7.

This paper provides an excellent overview of the emerging problem of nosocomial infections and the problems caused by antimicrobial resistance. It offers strategies for preventing the emergence and spread of resistant bacteria within the hospital environment.

9. Tomasz A. Multiple-antibiotic-resistant pathogenic bacteria. A report on the Rockefeller University workshop. N Engl J Med 1994;330:1247-51.

This paper is the result of a workshop held at Rockefeller University to discuss the emerging threat of antimicrobial resistance. It provides an overview of the problem, prospects for the future and recommendations to prevent further spread.

\section{LAY SUMMARY}

Antibiotic resistance of bacteria that cause infections in children has become a major problem over the past several years. Consequently, many infections cannot be treated with 'traditional' antibiotics and newer (often more expensive) drugs must be used. There are many reasons why bacteria develop resistance to antibiotics, and some measures can be taken to prevent the problem from becoming more serious. One of the most serious causes of antibiotic resistance is inappropriate use. Antibiotics may kill some, but not all bacteria. Consequently, bacteria naturally resistant to that antibiotic can be selected out and flourish. This problem is heightened when antibiotics are used for the wrong reasons or for an incorrect length of time. For instance, a child with a 'cold' is infected with a virus, for which there is no effective antibiotic. Nonetheless, antibiotics are often prescribed for such children; their use does not cure the infection and can encourage development of antibiotic resistance among bacteria that normally inhabit that child's throat or intestinal tract. If that child subsequently develops a bacterial infection, the bacteria may be resistant to the antibiotic used to treat the cold. This is but one of many examples of how the inappropriate use of antibiotics can result in infection with antibiotic-resistant bacteria.

What can you do? Everyone can become involved in the fight against antibiotic resistant bacteria. To protect yourself and your family against such infection, you should take the following steps:

1. Try to ensure that your child only receives antibiotics when they clearly are indicated for treatment of a bacterial infection. Children with colds should not receive antibiotic therapy.

2. Be sure that your child receives antibiotics at the prescribed dose and continues taking them for the duration prescribed.

3. Be sure that your child is fully immunized. Prevention of infection is the best way to fight antibiotic resistance.

4. Maintain the highest possible level of cleanliness in your home and in your child's daycare centre. Infections can be readily spread among children, particularly those who are in diapers and those who are in close contact. 


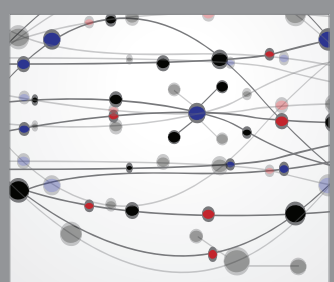

The Scientific World Journal
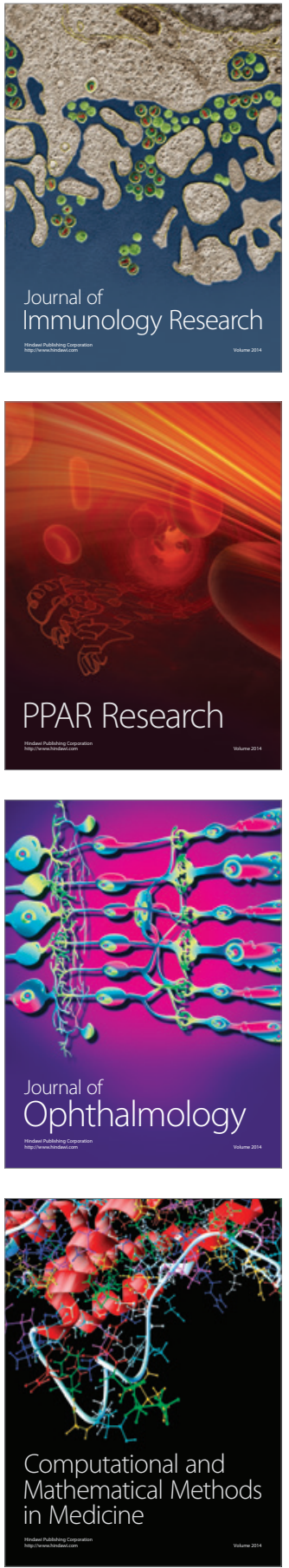

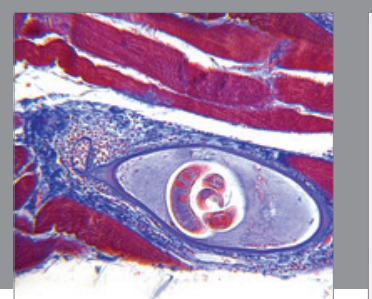

Gastroenterology Research and Practice

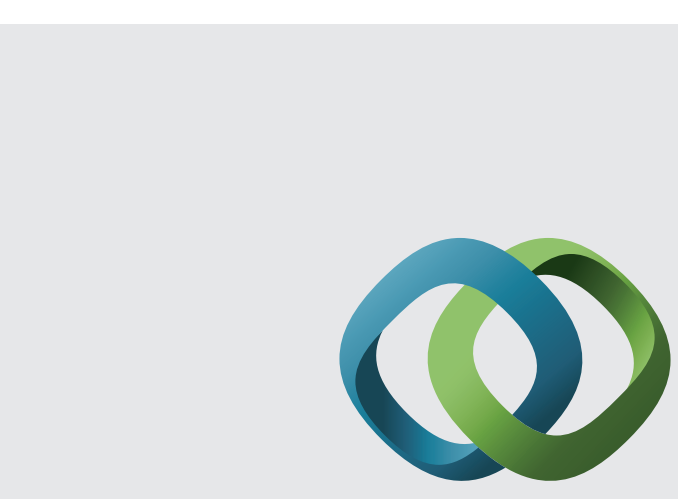

\section{Hindawi}

Submit your manuscripts at

http://www.hindawi.com
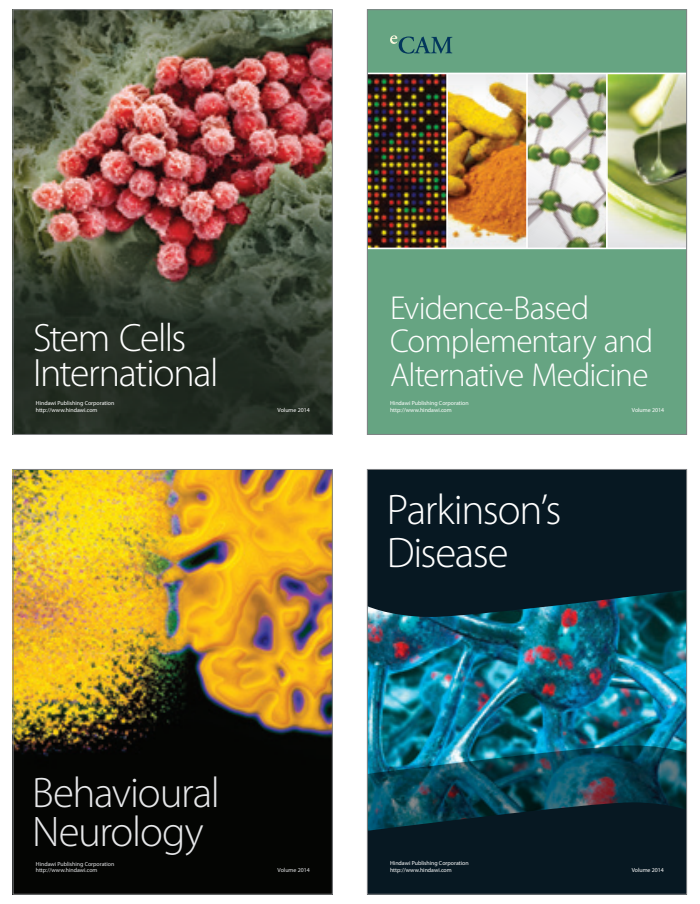
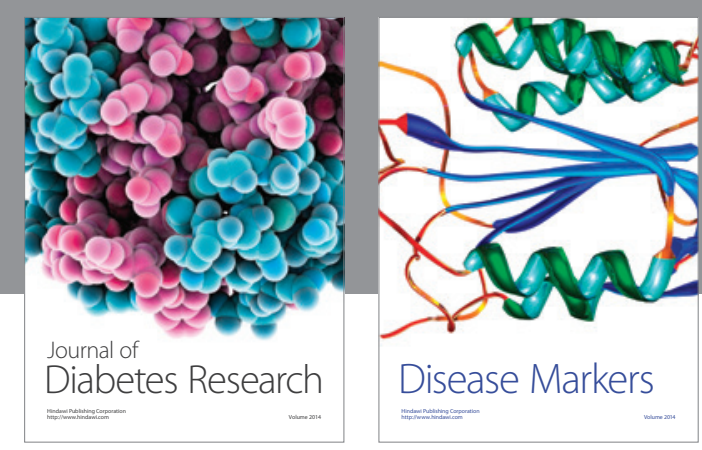

Disease Markers
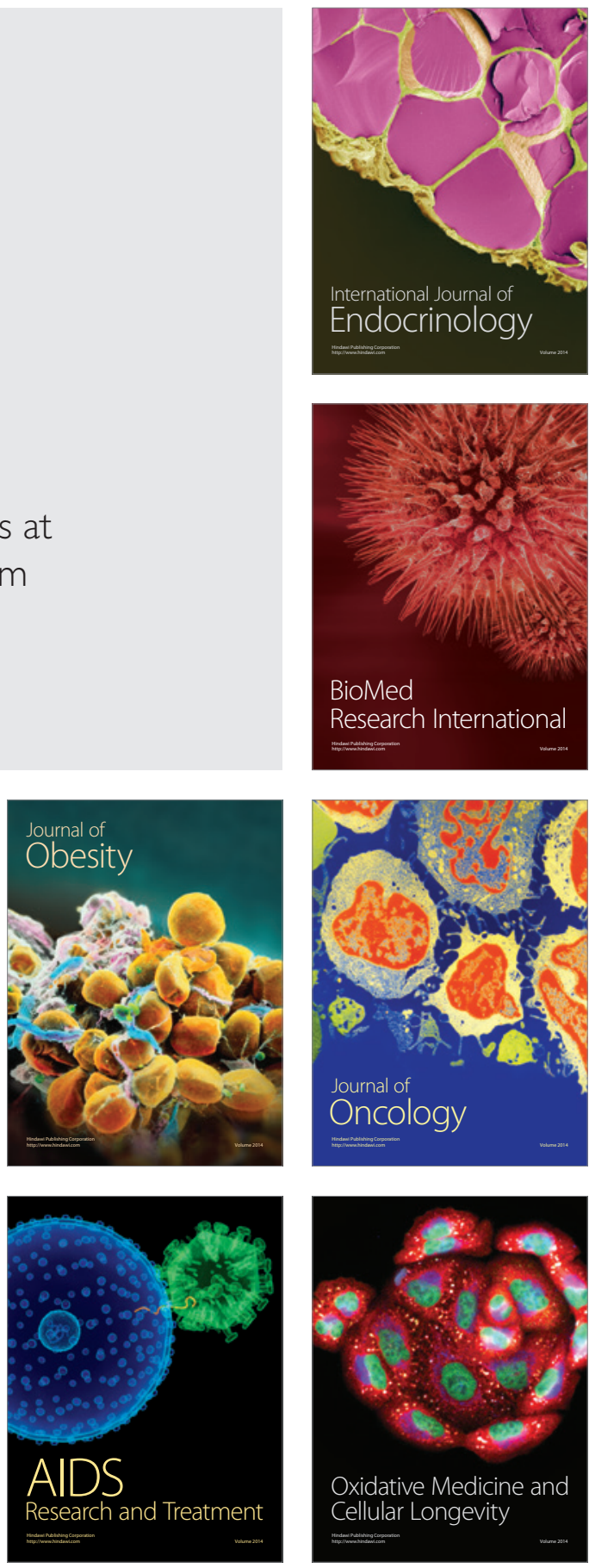